\section{Inverse Methods and Relative Nuclear Radii}

\author{
E. F. Hefter*,** and I. A. Mitropolsky*
}

Z. Naturforsch. 39a, 603-604 (1984);

received March 30, 1984

Inverse methods are applied to the nuclear bound-state problem. Considering only the self-interactions of these states analytical solutions results for potentials and densities. The simplest possible approximation to the full expression yields immediately $\Delta R_{0 i}^{2} \equiv\left\langle r^{2}\left(A_{i}\right)\right\rangle-\left\langle r^{2}\left(A_{0}\right)\right\rangle$ $\sim-\left[B\left(A_{i}\right)-B\left(A_{0}\right)\right]$ for the differences in the squared nuclear radii as functions of the respective binding energies per nucleon, $B\left(A_{i}\right)$.

\section{Motivation}

In contrast to their general usage we applied recently [1] inverse methods to the nuclear bound-state problem and not to scattering problems. Inverse methods solve the levels to the total density $\varrho(x) \equiv \sum \varrho_{i}(x) \equiv \sum \psi_{i}^{2}(x)$ where the very r.h.s. holds for the real potentials we are dealing with (see below). The characteristic feature of the individual $U_{N i}$ (and $\varrho_{i}$ ) is their specific amplitude-argument interdependence, i.e. the amplitude $U_{N i}(0)\left(\varrho_{i}(0)\right)$ appears again in the respective formfactor $f(x)(g(x))$.

The aim of this note is to show that this peculiar behaviour leads already to some useful predictions related to relative nuclear (charge rms) radii.

\section{Inverse Methods}

Inverse methods have been applied [1] to solve the $N$ single-particle Schrödinger equations approximating the nuclear many-body problem. To arrive at expressions that can be handled analytically, it is sensible to consider for the time being only spherically symmetric systems. Hence, the 3D Schrödinger equations may (with the appropriate care and boundary conditions, e.g. $\left.\varphi_{n}(r) \equiv \psi_{n}(x) / x ; x \geqq 0\right)$ be reduced to the corresponding (radial) 1D Schrödinger equations

$$
-M \partial_{x x} \psi_{n}(x)+\mathcal{L}(x) \psi_{n}=E_{n} \psi_{n} ; \quad M \equiv \hbar^{2} / 2 m ; \quad \partial_{x x} \equiv \partial^{2} / \partial x^{2} ; \quad n=1,2, \ldots, N ; \quad E_{n}<0 .
$$

The general solution of this inverse problem can only be obtained numerically. However, if we include only the contributions (self-interactions) of the $N$ occupied ground-state levels in our considerations (i.e. if we suppress completely possible contributions from the continuum), then we arrive at the well-known [1,2] analytical Bargmann potentials (or for 1D problems: reflectionless potentials):

$$
\begin{aligned}
& U_{N}(x)=\sum_{i=1}^{N} U_{N i}(x)=\sum_{i=1}^{N}\left[-4 \sqrt{-E_{i} M}\right] \varrho_{i}(x)=\sum_{i=1}^{N}\left[-4 \sqrt{-E_{i} M}\right] \cdot \psi_{i}^{2}(x)=-2 M \partial_{x x} \ln (\operatorname{det}(F)), \\
& F_{i j}=\delta_{i j}+2 \sqrt{f_{i}(x) f_{j}(x)} /\left(\sqrt{-E_{i}}+\sqrt{-E_{j}}\right) ; \quad f_{i}(x)=\sqrt{-E_{i}} \cdot \exp \left(\sqrt{-2 E_{i} / M} \cdot x\right) .
\end{aligned}
$$

Schrödinger problem in an equally respectable way as the traditional direct approach. But the required input is now given by information related to the energy spectrum of the Schrödinger operator from which the respective (scattering or) shell-model potential is evaluated.

In previous applications [1] it has been discussed that the complete expression for the shell-model potential with its $N$ bound states, $U_{N}$, may formally be written as the sum of the contributions from the individual states, the $U_{N i}$ (for the interrelation $x \leftrightarrow r$ see below):

$$
\begin{aligned}
U_{N}(x) & =\sum_{i=1}^{N} U_{N i}(x)=\sum_{i=1}^{N} U_{N i}(0) \cdot f\left(x ; U_{N i}(0)\right) \\
& =\sum_{i=1}^{N} a_{i} \varrho_{i}(x)=\sum_{i=1}^{N} a_{i} \varrho_{i}(0) g\left(x ; \varrho_{i}(0)\right) .
\end{aligned}
$$

The $a_{i}$ are state-dependent weighing coefficients and the $\varrho_{i}(x)$ are the contributions of the $i$ occupied ground-state

\footnotetext{
* Leningrad Nuclear Physics Institute, Gatchina/Leningrad, USSR.

** Institut für Theoretische Physik, Universität Hannover, Appelstrasse 2, 3000 Hannover 1, FRG ***.

*** Permanent address.
}

Reprint requests to E. F. Hefter, Institut für Theoretische Physik, Universität Hannover, Appelstrasse 2, 3000 Hannover 1 , FRG.
Inspection of (3) shows that wavefunctions, densities and potential are uniquely specified by the $N$ energy eigenvalues $E_{i}$ (which can, at least in principle, be taken from experiment). As indicated above, each of the individual contributions $Q_{i}(x)$ to the total density $\varrho(x)$ has the peculiar property that its amplitude appears again in its formfactor. Since this feature seems to carry the signature of the complete expression, it is tempting to use it in an attempt to approximate a nucleus by $U_{1}$ instead of using the more appropriate $U_{N}$. However, if we do so, then it is no longer sensible to use a single energy eigenvalue $E_{i}$ in $U_{1}$; we should rather take an "appropriately" weighed average over the energy eigenvalues of the respective nucleus. In view of the close relation, $B(A) \cong(0.35 / A)$ - $\sum n_{i} E_{i}$, between the binding energy per nucleon, $B(A)$, of a nucleus containing $A$ nucleons and its energy eigenvalues $E_{i}$ (the $n_{i}$ are the degeneracies: $n_{i} \equiv 2 j+1$ ) [3] it appears most reasonable to use the binding energies per nucleon, $B(A)$, for that purpose. With this substitute for $E_{i}$ the respective expression for $U=U_{1}$ reads (suppressing the index "1"):

$$
\begin{aligned}
U(x) & =-U_{0} \cdot \operatorname{sech}^{2}\left(\sqrt{U_{0} / 2 M} \cdot x\right) \\
& =2 B(A) \cdot \operatorname{sech}^{2}(\sqrt{-B(A) / M} \cdot x) \\
& =-4 \sqrt{-B(A) M} \cdot \varrho_{0} \cdot \operatorname{sech}^{2}\left(2 \varrho_{0} x\right)
\end{aligned}
$$

with $B(A)<0$ and $\varrho_{0} \equiv \sqrt{-B(A) / 4 M}$. For large (small) values of the argument the sech ${ }^{2}$-formfactor is very close to the more familiar Woods-Saxon (Gaussian) one as may be

0340-4811 / 84 / 0600-0603 \$ 01.3 0/0. - Please order a reprint rather than making your own copy. 
verified by the aid of the series expansion of the sech. Hence, it does make sense to approximate a complete nucleus by (4).

From (4) we readily obtain the half-density at halfwidth radius,

$$
\begin{aligned}
R(B) & =\left(1 / 2 \varrho_{0}\right) \cdot \operatorname{arccosh}(\sqrt{2})=0.4406 / \varrho_{0} \\
& =0.4406 \sqrt{-4 M / B(A)} ; \quad \varrho_{0} \equiv \sqrt{-B(A) / 4 M} .
\end{aligned}
$$

Since $M$ is a constant, see (2), and since the $B(A)$ are known to a good accuracy [4], (5) contains no adjustable parameters. However, since (5) does in no way account for the global changes in the absolute radii, which are well known to follows roughly an $A^{1 / 3}$ law, it can only yield estimates related to relative nuclear radii (and not to absolute radii). The quantity traditionally [5-7] referred to in the discussion of relative nuclear (charge $\mathrm{rms}$ ) radii is

$$
\begin{aligned}
\Delta R_{0 i}^{2} & \equiv\left\langle r^{2}\left(A_{i}\right)\right\rangle-\left\langle r^{2}\left(A_{0}\right)\right\rangle \\
& =\left[0.7765 \mathrm{M} / B\left(A_{0}\right) B\left(A_{i}\right)\right] \cdot\left[B\left(A_{i}\right)-B\left(A_{0}\right)\right] N_{B} .
\end{aligned}
$$

where the r.h.s. is due to (5); but for the renormalization constant $N_{B}$ which has been added "by hand".

\section{Discussion}

The constant $N_{B}$ is obviously the only adjustable parameter in (6). Hence, the resulting numbers are predictions for the behaviour of relative nuclear radii as functions of the mass numbers and/or their binding energies. However, even before attempting a comparison of the predictions of (6) with experiment, we know that it can not be a universal relation:

From the averaged input to be used, i.e. the $B(A)$ instead of all the $E_{i}$ in $U_{N}$, and from the restriction to spherically symmetric problems one realizes immediately certain limitations, i.e. (i) the existence of deformed nuclei is ignored, (ii) shell-effects and pairing correlations are almost completely suppressed, (iii) (6) does not distinguish between mass and charge distributions.

[1] E. F. Hefter and K. A. Gridnev, Z. Naturforsch. 38a, 813 (1983); E. F. Hefter, Proc. Workshop on Semiclass. Meth. in Nucl. Phys. to appear in Journal de Phys.Coll. series of J. de Physique (Sept. 1984) and Phys. Lett. B (in press); and references.

[2] E. B. Plekhanov, A. S. Suzko, and B. N. Zakhariev, Ann. Physik Berlin 39, 313 (1982); C. Quigg and J. L. Rosner, Phys. Rev. D23, 2625 (1983); P. Asthana and A. N. Kamal, Z. Physik C19, 37 (1983); and references.

[3] E. F. Hefter and I. A. Mitropolsky, LNPI-Report-860, Leningrad Nucl. Phys. Inst., Gatchina/Leningrad 1983 and to be published.
Already 15 years ago Gerstenkorn [5] proposed a relation corresponding to (6), but for the denominator $B\left(A_{0}\right) B\left(A_{i}\right)$. Subsequently (see e.g. [6, 7] and references) it has been shown to be highly useful even allowing for specific predictions [6] which were later on confirmed by experiment. But according to $[6,7]$ there exists up to now no theory or model that provides a derivation of this relation. - For a given isotrope sequence with $A_{0} \gg 1$ we have $A_{0}-A_{i} \ll A_{0}$ and therefore $B\left(A_{0}\right) \cdot B\left(A_{i}\right)=B\left(A_{0}\right)$ - $\left(B\left(A_{0}\right)+\varepsilon_{i}\right) \cong$ const with $\varepsilon_{i} \ll B\left(A_{0}\right)$ as is readily verified by the aid of the tabulated experimental data displayed in [4]. Hence, application of inverse methods is seen to lead almost immediately to a theoretical confirmation of this (hitherto heuristic) relation. For applications [5-7] could be consulted so that we refrain from giving additional examples.

\section{Summary}

Summarizing, we drew attention to the specific amplitude-argument interdependence of the individual contributions $Q_{i}$ of the different states to the total density $\varrho$. It yields immediately a formal justification for the wellknown empirical linear relation between nuclear radii and binding energies. From the way in which (6) has been derived, it is obvious that it has to fail whenever deformations and/or shell-effects are important. Further investigations attempting to obtain a more detailed picture are still in progress.

\section{Acknowledgements}

E. F. H. is grateful to the Academy of Sciences of the USSR for supporting his stay at the LNPI in Gatchina and he appreciates the warm hospitality extended to him during that visit (1983). He would also like to thank K. Heilig and $\mathrm{H}$. Hühnermann for illuminating discussions on the properties of relative radii and on isotope shift measurements.

[4] A. H. Wapstra and K. Bos, At. Data Nucl. Data Tabl. 19, 177 (1977)

[5] S. Gerstenkorn, C.R. Acad. Sci. Paris 168 B, 1636 (1969).

[6] S. Gerstenkorn, Comments Atomic Mol. Phys. 9, 1 (1979).

[7] R. Wenz, E. Matthias, H. Rinneberg, and F. Schneider, Z. Physik A295, 303 (1980). 